\title{
Characterization of random Gaussian and non-Gaussian stress processes in terms of vibration fatigue
}

\author{
Bruno Colin ${ }^{*}$ \\ Nexter Systems, 11 Allée des Marronniers, 78022 Versailles Cedex, France
}

Received: 12 December 2016 / Accepted: 30 November 2017

\begin{abstract}
In the field of military land vehicles, random vibration processes generated by all-terrain wheeled vehicles in motion are not classical stochastic processes of a stationary and Gaussian nature. The non-Gaussian nature of the processes is expressed in particular by very significant flattening levels that can affect the fatigue design of mechanical structures, conventionally acquired by spectral approaches, based essentially on spectral moments of stress processes. Due to these technical considerations, techniques for the characterization of random excitation processes generated by this type of driving situation need to be developed, by proposing innovative characterization methods no longer based on deterministic spectral and/or temporal approaches but on temporal approaches of a stochastic nature. Indeed, to characterize the fatigue damage produced by non-stationary and non-Gaussian random processes, the author shows that it is now necessary to mix time-counting techniques used in the field of vibration fatigue with those of the sampling statistics used in estimation theory. This approach makes it possible to extrapolate favorably over time the level of damage to structures, from a statistical perspective, when this extrapolation phase is in practice carried out deterministically. This technique, referred to as the disjoint block method (BDM), has been tested successfully in the context of component specification techniques from the reliability standpoint since 2010, and just recently integrated AFNOR standards.
\end{abstract}

Keywords: Random vibration / non-stationary and non-Gaussian random processes / disjoint block method (BDM) / spectral XRS and XFS approach / temporal SRX and SFX approach

\section{Introduction}

In the context of the design of structures to vibration fatigue, it is common to characterize the stationary random excitation processes by their acceleration power spectral density (PSD), within a range of relevant frequencies, generally covering the LF $[1-50 \mathrm{~Hz}]$ and MF domains $[50$ $500 \mathrm{~Hz}$ ], for the case of wheeled vehicles.

Then relying on the conventional assumptions of Gaussian signals, characterization methods of the average fatigue damage generated by these random acceleration processes may be used. This is the case of characterization techniques by the response spectra (RS) method, widely used by manufacturers in the civil and military sectors, as part of the development of design vibration specifications.

The RS technique consists in characterizing the severity of the excitation process through a simple mechanical model consisting of a linear system with one degree of freedom (dof) whose natural frequency is varied $f_{0}$ within a sufficiently wide domain covering the natural frequencies of the structure to size, and limited by the traditional criterion

\footnotetext{
* e-mail: b.colin@nexter-group.fr
}

of the effective masses. In many industrial applications, the criterion of effective masses consists in retaining all the modes in which the sum of the effective masses is $90 \%$ of the total mass of the structure to size [1]. At this stage, it is a generalization of the Biot model, historically used in the calculation of the shock response spectrum (SRS) for transient excitations and that is adapted to the case of random vibration [2] referred to as the fatigue damage spectrum (FDS). Thus, for a process of random vibration evaluated generally on a limited portion $T_{\text {ref }}$ of the total stress time $T_{t}$, it is possible to characterize its severity by applying either a spectral domain approach (stochastic approach) or a temporal domain approach (deterministic approach), each of which has disadvantages in the case of non-Gaussian random processes, as discussed below.

\section{Selected standard system}

When a vibrational excitation process $\ddot{x}(t)$ is applied to the base of a mechanical system with one dof (mass - spring damper) the standard system responds by generating a relative movement $z(t)$ between the mass and the base of the standard system (see Fig. 1). This relative movement is 


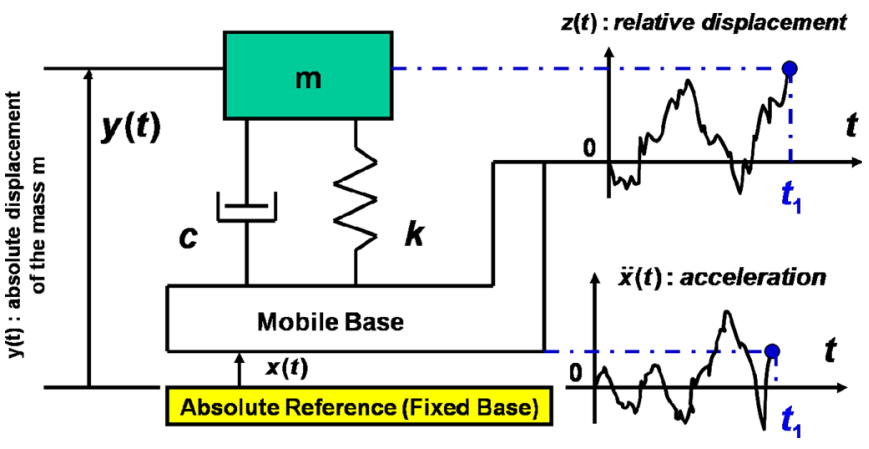

Fig. 1. Standard system with one dof.

then dependent on the natural frequency $f_{0}$ of the standard and is proportional to the uniaxial stress state $\sigma(t)$ generated in the structure, provided that the latter is considered linear (an assumption commonly used in design). The proportionality constant $K$ (Stress - Relative displacement) is imposed by the designer and is kept constant regardless of the value of the natural frequency $f_{0}$.

Therefore we obtain:

$$
\sigma(t)=K . z(t)
$$

where $\sigma(t)$ : amplitude of the vibration stress $\left(N / \mathrm{m}^{2}\right), K$ : proportionality constant $\left(\mathrm{N} / \mathrm{m}^{3}\right)$ between stress and relative displacement ( $K$ is a constant if it is assumed that the structure to be designed is linear), $z(t)$ : amplitude of the relative displacement $(m)$.

In which

$$
z(t)=y(t)-x(t)
$$

where $y(t)$ : absolute displacement of the mass $m(m), x(t)$ : absolute displacement of the mobile base $(\mathrm{m})$.

The standard system for mass $m$ (in $\mathrm{Kg}$ ), stiffness $k$ (in $N / \mathrm{m}$ ) and viscous damping $c$ (in N.s $/ \mathrm{m}$ ) is then characterized by its modal parameters, namely:

$$
\xi=\frac{c}{2 \cdot \sqrt{k \cdot m}} \quad \text { and } \quad Q=1 / 2 . \xi
$$

and

$$
f_{0}=\frac{1}{2 . \pi} \cdot \sqrt{\frac{k}{m}} \text { and } \omega_{0}=2 . \pi \cdot f_{0},
$$

where $\xi$ : critical or modal damping, $Q$ : modal overtension factor, $f_{0}$ : natural or modal frequency $(\mathrm{Hz}), \omega_{0}$ : natural or modal pulsation $(\mathrm{rd} / \mathrm{s})$.

The uniaxial stress state of the standard system is characterized through the standard system by assessing its response, in terms of relative displacement which is estimated either in frequency or temporal form, depending on whether a frequency or temporal domain approach is used to evaluate the fatigue damage associated with standard system in question.

\subsection{Frequency domain approach}

In the case of a frequency domain approach, the standard system is characterized by its transfer function
$H_{\ddot{x} z}(\omega=2 . \pi . f)$, defined between the acceleration of $\ddot{x}(t)$ the vibrational excitation and the relative displacement $z(t)$ of the response, which is then characterized by its noted unilateral Power Spectral Density $\operatorname{PSD}_{z z}(\omega=2 . \pi . f)$.

The expression of the standard system transfer function is then defined as follows:

$$
H_{\ddot{x} z}(\omega)=\frac{Z(\omega)}{\ddot{X}(\omega)}=\left|H_{\ddot{x} z}(\omega)\right| . e^{j . \angle H} \ddot{x} z^{(\omega)},
$$

where

$Z(\omega)=\operatorname{TF}[z(t)] \quad$ and $\quad \ddot{X}(\omega)=\operatorname{TF}[\ddot{x}(t)]$

$\left|H_{\ddot{x} z}(\omega)\right|:$ modulus of the Transfert Function

$\angle H_{\ddot{x} z}(\omega)$ : phase of the Transfert Function

TF[.]: $\quad$ Fourier Transform operator.

And :

$$
\left|H_{\ddot{x} z}(\omega)\right|=\frac{1}{\omega_{0}^{2}} \cdot \frac{1}{\sqrt{\left(1-h^{2}\right)^{2}+4 \cdot \xi^{2} \cdot h^{2}}}
$$

where $h=\frac{\omega}{\omega_{0}}=\frac{f}{f_{0}}, \quad \omega_{0}=\sqrt{\frac{k}{m}}=2 . \pi . f_{0}$ and $Q=1 / 2 . \xi$.

Due to these technical considerations, it is possible to simply assess the pace of unilateral PSD of the relative movement $\operatorname{PSD}_{z z}(\omega)$ in $\mathrm{m}^{2} / \mathrm{Hz}$, knowing the unilateral PSD of the excitation $\operatorname{PSD}_{\ddot{x} \ddot{x}}(\omega)$ in $\left(\mathrm{m} / \mathrm{s}^{2}\right)^{2} / \mathrm{Hz}$, based on the classic expressions of the dynamics of linear structures [3] namely:

$$
\operatorname{PSD}_{z z}(\omega)=\left|\mathrm{H}_{\ddot{x} z}(\omega)\right| \cdot \operatorname{PSD}_{\ddot{x} \ddot{x}}(\omega)
$$

where $\omega \in\left[\omega_{\min }, \omega_{\max }\right]$.

\subsection{Temporal domain approach}

In the case of a temporal domain approach, the standard system is characterized by its impulse function $h_{\ddot{x} z}(t)$, defined between the acceleration $\ddot{x}(t)$ of the vibrational excitation and the relative displacement $z(t)$ of the response, which is then characterized by its $z$-transform, $H_{\ddot{x} z}(z)$ within the meaning of the first order hold (FOH) method, used for the mathematical reconstruction of the response $z(t)$ sampled [4]. The expression of the $z$-transform of the impulse function of the standard system is then defined as follows [5]:

$$
\begin{aligned}
H_{\ddot{x} z}(z) & =\frac{(z-1)^{2}}{\Delta t . z} \cdot \mathrm{TZ}\left\{\mathrm{TL}^{-1}\left[\frac{H_{\ddot{x} z}(p)}{p^{2}}\right]\right\} \\
& =\frac{b_{0}+b_{1} \cdot z^{-1}+b_{2} \cdot z^{-2}}{1+a_{1} \cdot z^{-1}+a_{2} \cdot z^{-2}}
\end{aligned}
$$

where $\quad z=\exp (p . \Delta t)$ and $F_{e}=\frac{1}{\Delta t}$,

where $F_{e}$ : Sampling frequency of the random excitation process $\ddot{x}(t)$ and the response $z(t)$ in $\mathrm{Hz}$.

The advantage of using the $z$-transform instead of the impulse function basically stems from in the speed of the calculations of the $z(t)$ sampled response, which, as part of 
the process of characterizing the random non-Gaussian stress processes, is an important issue, as we shall see below with the notion of temporal XFS, explained below in Section 5.2.

Due to these technical considerations, it is then possible to simply evaluate the expression of the sampled response of the relative displacement $z(i . \Delta t)=z(i)$ over the reference duration $\mathrm{T}_{\text {ref }}$ corresponding to the acquisition time of the excitation processes $\ddot{x}(\mathrm{t})$ to be characterized in terms of vibration fatigue, namely:

$$
\begin{aligned}
z(i)= & -a_{1} \cdot z(i-1)-a_{2} . z(i-2)+b_{0} . \ddot{x}(i)+b_{1} . \ddot{x}(i-1) \\
& +b_{2} . \ddot{x}(i-2)
\end{aligned}
$$

where $i=[1, n]$ and $n=T_{\text {ref }} \cdot F_{e}$,

where $T_{\text {ref: }}$ acquisition time of the excitation processes $\ddot{x}(t)$ And:

\section{See equation 11 below page}

\section{Approaches for calculating FDS}

As presented in the introduction, there are two possible approaches for calculating the FDS of a stationary random excitation process, depending on whether we wish:

- to adopt the case of a stochastic approach to estimate the statistics of damage fatigue levels generated by the excitation process $\ddot{x}(t)$ that we wish to characterize favorably in terms of mean and $(1-\alpha)$ quantile value, or order quantile $(1-\alpha)$, for the total stress time during which $T_{t}$ the random excitation process is applied. This stochastic approach is especially important in that it can be used to extrapolate statistically fatigue damage levels over a $T_{t}$ longer $\left(T_{t}>T_{\text {ref }}\right)$ period than that associated with the reference time $T_{\text {ref }}$ for which the excitation process $\ddot{x}(t)$ is measured or estimated by modeling (as shown in Sect. 3.1)

- or adopt the case of a deterministic approach allowing only calculate the damage fatigue levels generated by the excitation process $\ddot{x}(t)$ acquired on the reference time $T_{\text {ref }}$ by measurement or numerical modeling, without being able to extrapolate favorably on the total duration $T_{t}$ other than by a deterministic approach to proportionality, as presented in Section 3.2. Therefore, it is clear that this method of temporal calculation can be non-conservative and can therefore lead to significant mechanical undersizing for the structures being studied. This is especially true if the duration $T_{\text {ref }}$ is short compared with the total duration $T_{t}$ of the stress in question.

Beyond the identified risk of undersizing in the case of temporal domain approaches, the latter lead to prohibitive computing time, unlike the spectral methods. Indeed, the advantage of spectral methods lies in the fact that the calculation of the FDS is analytical, but the associated upcrossing risk $\alpha$ should be made clear. For example, if one takes for simplicity the case of random stationary and Gaussian processes, it is possible to show that this notion of FDS is implicitly associated with an up-crossing risk which is not constant and equal to $50 \%$, as suggested by many designers and specifiers. Indeed the work of the author in 2007 [6] showed that the level of accumulated damage could favorably be described by a 2-parameter Weibull distribution $(\tilde{\beta}, \tilde{\sigma})$ and not by a normal distribution conventionally used by some authors. Due to these technical considerations, it is possible to show that the concept of spectral FDS is associated with an up-crossing risk, depending on the shape parameter $\tilde{\beta}$ of this Weibull distribution, which is then based on the calculation parameters of the Spectral FDS:

- $f_{0}$ and $Q$ : modal parameters of the standard system;

- $T_{t}$ : total duration of the vibratory stress $\ddot{x}(t)$ in question;

$-b$ : basquin slope of the fatigue behavior of the structure in question, described by equation (12) and the following Figure 2.

$$
N \cdot\left[\sigma_{a}\right]^{b}=C=A^{b},
$$

where $b$ and $C=A^{b}$ : material parameters of the structure in question, $A$ : alternating failure stress of the material $\left(N / \mathrm{m}^{2}\right), N$ : average number of failure cycles (cycles), $\sigma_{a}$ : sinusoidal alternating stress, corresponding to local extrema (maxima and/or minima) of the stress process $\sigma(t)=K \cdot z(t)$ defined by $(1)$ in $N / \mathrm{m}^{2}$.

The fluctuation of the exceedance threshold associated with the conventional expression of the spectral FDS, depending on the frequency $f_{0}$, is not very reassuring as a factor for designers who are in charge of complex, highperformance structures. It is also for these reasons that the model of the conventional spectral FDS, widely used for establishing vibration specifications [7], has been

$$
\begin{aligned}
& a_{1}=-2 \cdot \exp \left(-\xi \cdot \omega_{0} \cdot \Delta t\right) \cdot \cos \left(\omega_{d} \cdot \Delta t\right) \\
& a_{2}=\exp \left(-2 \cdot \xi \cdot \omega_{0} \cdot \Delta t\right) \\
& \text { where } \omega_{d}=\omega_{0} \cdot \sqrt{1-\xi^{2}} \text { and } Q=1 /(2 \cdot \xi) \text { and } F_{e}=1 / \Delta t \\
& b_{0}=\frac{1}{\omega_{0}^{3} \cdot \Delta t} \cdot\left\{2 \cdot \xi \cdot\left[\exp \left(-\xi \cdot \omega_{0} \cdot \Delta t\right) \cdot \cos \left(\omega_{d} \cdot \Delta t\right)-1\right]+\exp \left(-\xi \cdot \omega_{0} \cdot \Delta t\right) \cdot\left[\frac{\omega_{0}}{\omega_{d}} \cdot\left(2 \cdot \xi^{2}-1\right) \cdot \sin \left(\omega_{d} \cdot \Delta t\right)\right]+\omega_{0} \cdot \Delta t\right\} \\
& b_{1}=\frac{1}{\omega_{0}^{3} \cdot \Delta t} \cdot\left\{-2 \cdot \omega_{0} \cdot \Delta t \cdot \exp \left(-\xi \cdot \omega_{0} \cdot \Delta t\right) \cdot \cos \left(\omega_{d} \cdot \Delta t\right)-2 \cdot \frac{\omega_{0}}{\omega_{d}} \cdot\left(2 \cdot \xi^{2}-1\right) \cdot \exp \left(-\xi \cdot \omega_{0} \cdot \Delta t\right) \cdot \sin \left(\omega_{d} \cdot \Delta t\right)+2 \cdot \xi \cdot\left[1-\exp \left(-2 \cdot \xi \cdot \omega_{0} \cdot \Delta t\right)\right]\right\} \\
& b_{2}=\frac{1}{\omega_{0}^{3} \cdot \Delta t} \cdot\left\{\left(2 \cdot \xi+\omega_{0} \cdot \Delta t\right) \cdot \exp \left(-2 \cdot \xi \cdot \omega_{0} \cdot \Delta t\right)+\exp \left(-\xi \cdot \omega_{0} \cdot \Delta t\right) \cdot\left[\frac{\omega_{0}}{\omega_{d}} \cdot\left(2 \cdot \xi^{2}-1\right) \cdot \sin \left(\omega_{d} \cdot \Delta t\right)-2 \cdot \xi \cdot \cos \left(\omega_{d} \cdot \Delta t\right)\right]\right\} \cdot(11)
\end{aligned}
$$



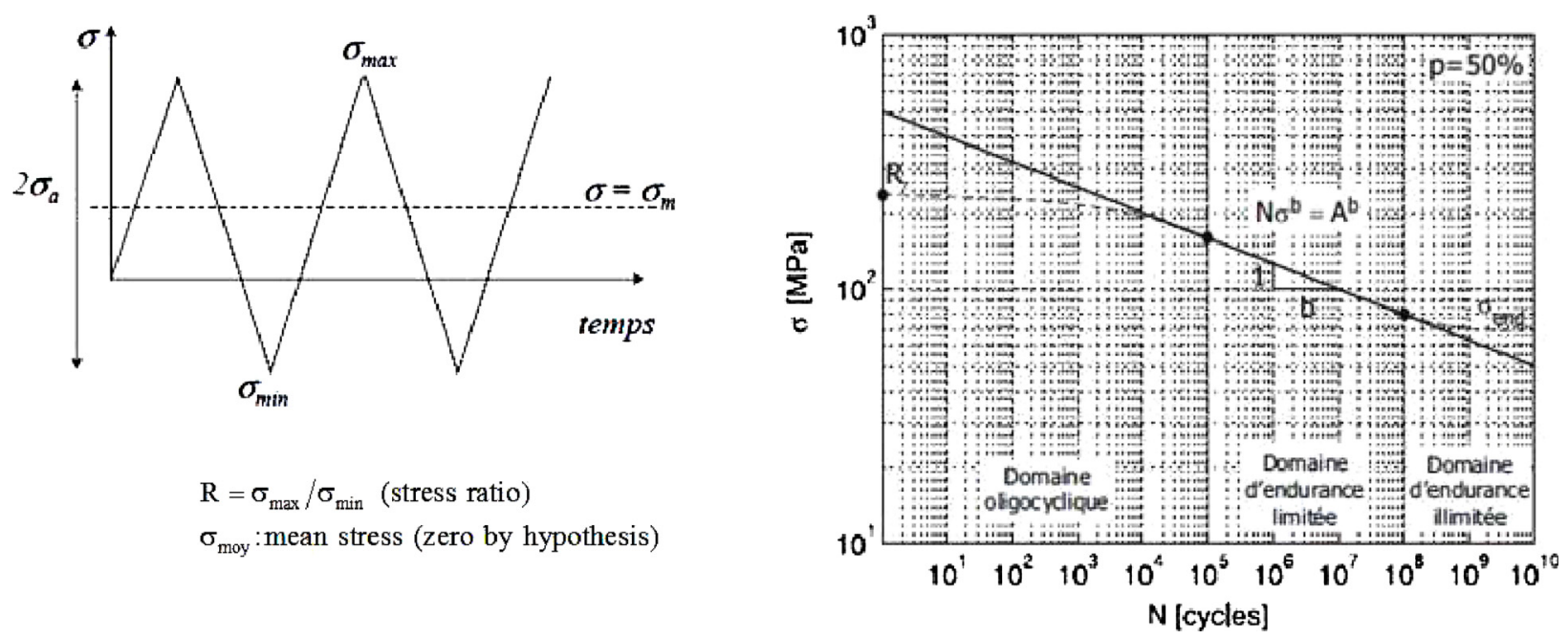

Fig. 2. Basquin model and convention of writing.

generalized by the spectral XFS approach, which is the counterpart of the spectral XRS approach also proposed in 2007 by the author [6].

\subsection{Calculation of the spectral FDS}

There are different possible approaches to the calculation of spectral FDS according to the counting method (Peak-Valley and/or Rainflow Counting [8]). In the present case, the one proposed here is based on the extrema counting method (Peak-Valley) and is associated with the following factors: - the excitation process $\ddot{x}(t)$ of duration $T_{t}$ is stationary, ergodic, Gaussian and has a zero mean (all the spectral moments are therefore constant and calculable from the PSD of the relative movement of the standard system); - the standard system adopted is linear, the relative displacement $z(t)$ of the model is itself stationary, ergodic, Gaussian and zero mean. In addition the standard system being a quality factor $Q \geq 3$, the random stress process has a narrow band centered on $f_{0}$ in which the local maxima are characterized by a Rayleigh distribution;

- the fatigue behavior of the structure is described by its Basquin's law, defined by its parameters $b$ and $C=A^{b}$ (see relation 12 above);

- the accumulated damage per cycle is based on Miner's conventional linear accumulation rule [9];

- only the positive local maxima and negative local minima of the stress process $\sigma(t)=K . z(t)$ are assumed to be damaging.

The spectral FDS is then calculated from the unilateral PSD of the random stress process $\sigma(t)$ which is assumed to be Gaussian and therefore from the random process associated with the relative displacement $z(t)$ of the standard system, insofar as the latter is considered here to be linear. For each natural frequency $f_{0}$, the value of the average cumulative damage $E\left[D_{c}(\xi)\right]$ is calculated from the damage produced by different cycles extracts over the total duration of $T_{t}$ the excitation process $\ddot{x}(t)$ in question and accumulated linearly through the fatigue behavior of the structure in question. Based on the above assumptions, it can be easily shown that the analytical expression of the spectral FDS of a Gaussian random process $\ddot{x}(t)$ can be calculated simply by using the Rayleigh distribution $f_{Z_{\max }}(z)$ associated with the random process of the local maxima of the relative displacement $z(t)$, i.e.:

$$
\begin{aligned}
& f_{Z_{\max }}(z)=\frac{z}{\sqrt{m_{0}}} \cdot \exp \left(-\frac{z^{2}}{2 \cdot m_{0}}\right), \text { where } z \in[0,+\infty] \\
& \text { and } m_{0}=z_{\mathrm{eff}}^{2}\left(f_{0}\right)=\int_{f=0}^{+\infty} \operatorname{PSD}_{\mathrm{zz}}(f) \cdot d f
\end{aligned}
$$

$\operatorname{PSD}_{z z}(f)$ : unilateral PSD of the random process $z(t)$.

Hence the conservative expression of the (narrow-band) spectral FDS is: $\operatorname{FDS}_{\mathrm{sp}}^{\mathrm{BE}}\left(f_{0}\right)$

$\operatorname{FDS}_{\mathrm{sp}}^{\mathrm{BE}}\left(f_{0}\right)=\left\{\begin{array}{l}\frac{C}{K^{b}} \cdot E\left[D_{c}(\xi)\right]=n \cdot \int_{\mathrm{z}=0}^{+\infty} z^{b} \cdot f_{Z_{\max }}(z) \cdot d z \\ \left(f_{0} \cdot T_{t}\right) \cdot\left[2 \cdot z_{\mathrm{eff}}^{2}\left(f_{0}\right)\right]^{b / 2} \cdot \Gamma\left(1+\frac{b}{2}\right)\end{array}\right.$

where $n=f_{0} \cdot T_{t}$.

This simple expression of the narrow-band spectral FDS is conservative and can be generalized to the case of broad-band Gaussian processes, relying on the Rice Theory of stationary random Gaussian processes $[10,11]$. However, the disadvantage of these spectral methods is that they remain subject to a restrictive assumption of gaussianity, which now needs to be abandoned, in order to positively expand the scope of the RS method.

\subsection{Calculation of the temporal (deterministic) FDS}

In the case of the temporal FDS, the relative displacement $z(t)$ is obtained by the FOH method presented in Section 2.2 above and this for $t \in\left[0, T_{\text {ref }}\right]$, as the excitation $\ddot{x}(t)$ is 
known only for the reference period $T_{\text {ref }}$. The absolute values of the peaks (positive maximum) and valleys (negative minimum) of the relative displacement $z(t)$ evaluated over time $T_{\text {ref}}$, are determined using the conventional counting method of extrema which associates with a maximum or minimum a half cycle of damage to stay in the same calculation assumptions as the spectral FDS. This "Peak-Valley" method can then be used to retrieve from the relative displacement $z(t)$ of duration $T_{\text {ref: }}$ :

- the $n_{\max }$ damage half-cycles associated with the positive maximum amplitude $Z_{\max }(i)$ for $i=1$ a $n_{\max }$;

- the $n_{\min }$ damage half-cycles associated with the negative minimum amplitude $Z_{\min }(i)$ for $i=1$ a $n_{\min }$.

The half cycles thus retrieved from the random stress process $\sigma(t)=K \cdot z(t)$, defined on the reference duration $T_{\text {ref }}$ are then accumulated linearly in terms of partial damage $D_{p}$ using Basquin's law defined by (12) and Miner's rule defined as follows:

$$
\begin{aligned}
D_{p} & =\sum_{j=1}^{n_{\max }} d_{\max }(j) \\
& +\sum_{j=1}^{n_{\min }} d_{\min }(j) \text { avec }\left\{\begin{array}{l}
d_{\max }(j)=\frac{K^{b}}{2 \cdot C} \cdot\left[Z_{\max }(j)\right]^{b} \\
d_{\min }(j)=\frac{K^{b}}{2 \cdot C} \cdot\left[\left|Z_{\min }(j)\right|\right]^{b}
\end{array}\right.
\end{aligned}
$$

The extrapolation in the temporal domain of this partial damage over the total stress duration $T_{t}$ is performed in a deterministic manner, using the following rule of proportionality; and can therefore be used to calculate the cumulative damage $D_{c}$, respecting the same assumptions used for the calculation of the average cumulative damage $E\left[D_{c}(\xi)\right]$ :

$$
\begin{aligned}
D_{c} & =\frac{T_{t}}{T_{\text {ref }}} \cdot D_{p} \\
& =\frac{T_{t}}{T_{\text {ref }}} \cdot \frac{K^{b}}{2 \cdot C} \cdot\left[\sum_{j=1}^{n_{\max }}\left[Z_{\max }(j)\right]^{b}+\sum_{j=1}^{n_{\min }}\left[\left|Z_{\min }(j)\right|\right]^{b}\right] .
\end{aligned}
$$

This calculation procedure is then applied to all of the systems with one dof in question for each of the natural frequencies $f_{0}$, which leads to the following expression of the temporal FDS, noted $\operatorname{FDS}_{t p}^{D}\left(f_{0}\right)$, of a deterministic nature:

$$
\begin{aligned}
\operatorname{FDS}_{t p}^{D}\left(f_{0}\right) & =\frac{C}{K^{b}} \cdot D_{c} \\
& =\frac{T_{t}}{2 \cdot T_{\text {ref }}} \cdot\left[\sum_{j=1}^{n_{\max }}\left[Z_{\max }(j)\right]^{b}+\sum_{j=1}^{n_{\min }}\left[\left|Z_{\min }(j)\right|\right]^{b}\right] .
\end{aligned}
$$

Note: As in the case of the SRS [12] and the temporal ERS, it is recommended here to sample the random excitation process $\ddot{x}(t)$ at a frequency at least 10 times greater than the maximum frequency of the analysis of the RS in question, in order to correctly evaluate the temporal FDS.

This technique of calculating the temporal FDS of a deterministic nature, although very easy to develop from a numerical point of view, is very often inadequate for the secure sizing of structures, which necessarily requires taking into account the statistics of the cumulative damage of stress processes of a stochastic nature. This therefore necessarily requires the use of probabilistic rather than deterministic methods, that are capable of extrapolating the values of the $(1-\alpha)$ quantiles of cumulative damage for a given total stress duration $T_{t}$.

\section{Securing structures and design risk control}

To secure the fatigue design of mechanical structures subjected to stationary random processes of prolonged stress $T_{t}$ we should now focus on the statistical characteristics of the accumulated fatigue damage levels generated by these processes over time $T_{t}$, applying a risk of exceeding them $\alpha$, which is determined according to the criticality level of the structure to be sized.

In practice, the three levels of risk, commonly used in the mechanical design of military land vehicles, are: $-\alpha=10 \%$ : Risk associated with the development of equipment and/or mechanical structures "common", not part of a functional chain of important performance for the end customer. This is particularly true of the components that are associated with secondary functions of the System;

$-\alpha=1 \%$ : Risk associated with the development of equipment and/or mechanical structures "standards", part of a functional chain of important performance (sensitivity) for the end customer, but the level of customer demand remains moderate. This is the case of the components that are associated with the main functions of the system, for which the functional performance levels specified by the end customer are achievable, safe, including the vibratory stresses taken into account in the development;

$-\alpha=0,1 \%$ : Risk associated with the development of "secure" mechanical equipment and/or structures as part of a functional chain of performance which is important for the end user, but for which the level of customer demand is high. This is the case of the components that are associated with the main functions of the system, for which the high functional performance levels specified by the end customer can only be achievable, without risk, if the stresses taken into account in the development are fully controlled.

Due to these technical considerations, it was therefore considered important in the years 2005-2010 [6,13] to propose major changes, to the RS techniques, to better understand the risks of exceeding vibration fatigue damage levels in the case of the excitation processes generated by military land vehicles. This work was first written in the context of random Gaussian processes, which helped to establish the notion of spectral XFS with an up-crossing risk $\alpha$, which replaces the case of spectral FDS, by definition associated with the accrued level of damage.

In Section 5 below, we therefore recall the main results of this notion of spectral XFS, which is subject to the case of Gaussian processes. It will be completed positively by innovative approaches that can be used to propose concepts of temporal XFS, which are now capable of taking into account the non-Gaussian nature of the random processes 

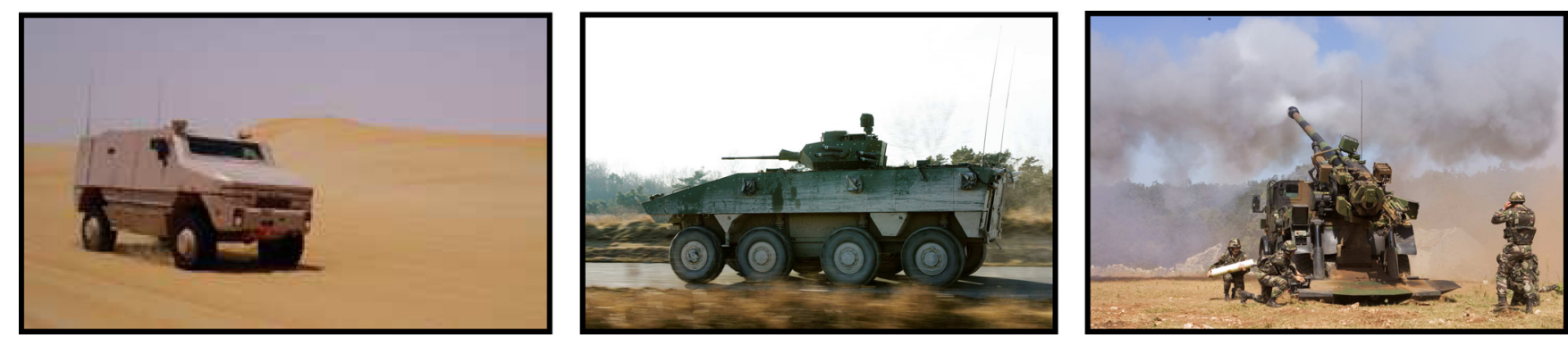

Fig. 3. ARAVIS $(4 \times 4$ of 12,5 Tons), VBCI $(8 \times 8$ of 29 Tons) and CAESAR (Artillery of 155 mm, with carriers $6 \times 6$ RTD or UNIMOG).

generated by Land vehicles. These techniques have been favorably used in the ARAVIS, VBCI and CAESAR vehicles, which are products developed by Nexter Systems on wheeled mobility platforms (see Fig. 3).

\section{XFS computing approach}

As presented above, it is essential now to estimate the statistical model of cumulative damage levels associated with random stress processes over the entire duration $T_{t}$ of the excitation process, regardless of the statistical nature of the excitation process (Gaussian or non-Gaussian). This results in no longer promoting the use of deterministic temporal FDS techniques, which seemed to be widespread among designers, since they did not have probabilistic methods suitable for the target technical issue. Indeed, the objective hitherto sought is to assess the threshold level of cumulative damage $\left[(1-\alpha)\right.$-quantile of the random variable $\left.D_{c}\right]$ over the total excitation period $T_{t}$ with a constant up-crossing risk $\alpha$ for each natural frequency $f_{0}$ imposed by the designer and/or specifier; whereas in the case of spectral FDS (see Sect. 5.1), this risk $\alpha$ is a function of the frequency values $f_{0}$ retained.

These up-crossing risk RS $\alpha$ were designated XFS in order to differentiate them from the term FDS and now cover the case of Gaussian and/or non-Gaussian stationary random processes, as presented below.

\subsection{Calculation of spectral XFS}

Based on the work conducted in 2007 by the author on a conservative approach associated with the case of stationary, zero mean and narrow-band Gaussian random processes, an analytical approach of the spectral XFS was established on the basis of the following assumptions, which are complementary to those defined for calculating the spectral FDS:

- the excitation process $\ddot{x}(t)$ of duration $T_{t}$ is stationary, ergodic, Gaussian, zero mean and broadband of the white noise type (a highly restrictive assumption and not consistent with the physical reality of Defense sector land vehicles);

- the damping level $\xi$ the standard system retained is weak $(\xi \leq 0,05)$ corresponding to a quality factor $Q \geq 10$;

- the numbers of stress cycles associated with the various standard systems are high and must respect the following order relation, namely: $\left(\xi \cdot f_{0} \cdot T_{t} \geq 1\right)$;
- the cumulative damage distribution law $D_{c}(\xi)$ is assumed to be represented by a 2-parameter Weibull distribution $(\tilde{\beta}, \tilde{\sigma})$, in which $\tilde{\beta}$ and $\tilde{\sigma}$ respectively represent the shape parameter and the scale parameter of Weibull distribution in question.

Indeed, insofar as the cumulative damage $D_{c}(\xi)$ is a positive support random variable, it is clear that the statistical model to retain cannot be Gaussian as claimed by some authors. The choice of a Weibull model has been preferred, given its exponential decay, which is the same as that associated with the Rayleigh model characterizing the local maxima of the narrow-band random stress process in question. Thus, based on these assumptions, the coefficient of variation of the cumulative damage $D_{c}(\xi)$ can be calculated analytically and leads to the following approximate result, namely [14]:

$$
\mathrm{CV}\left[D_{c}(\xi)\right] \approx\left[\frac{2 \cdot Q \cdot f_{1}(b)}{n}\right]^{1 / 2} \text { where } n=f_{0} \cdot T_{t}
$$

where $f_{1}(b)=\exp \left[c_{2} \cdot b^{2}+c_{1} \cdot b+c_{0}\right]$ for $b \in[3,20]$ and $c_{2}=$ $2,51419 E-03, c_{1}=5,04776 E-01, c_{0}=-2,38747 E+00$.

The shape of the function $f_{1}(b)$ is described in Figure 4 and is an approximate model of the infinite series from which it derives [6].

Due to these technical considerations, it appears that the 2-parameter Weibull model $(\tilde{\beta}, \tilde{\sigma})$ for the cumulative damage is now adjustable by the method of moments, by endeavoring to readjust for each standard system, the average value of cumulative damage provided by equation (14) and its coefficient of variation provided by the previous equation (18). Therefore, it is shown that the spectral XFS with up-crossing risk $\alpha$ can be calculated from the classical spectral FDS, using the following simple expression:

$$
\frac{\mathrm{XFS}_{\mathrm{sp}}^{\mathrm{BE}}\left(f_{0}\right)}{\operatorname{FDS}_{\mathrm{sp}}^{\mathrm{BE}}\left(f_{0}\right)}=\frac{[\ln (1 / \alpha)]^{\tilde{\lambda}}}{\Gamma(1+\tilde{\lambda})}=g_{4}(\alpha, b, Q, n)
$$

where $\tilde{\lambda}$ is such as $h(\tilde{\lambda})=0$

where $\quad h(\lambda)=\frac{\Gamma(1+2 . \lambda)}{\Gamma^{2}(1+\lambda)}-\mu \quad$ and $\quad \mu=\frac{2 . Q \cdot f_{1}(b)}{n}+1$

where $n=f_{0} \cdot T_{t}$. 


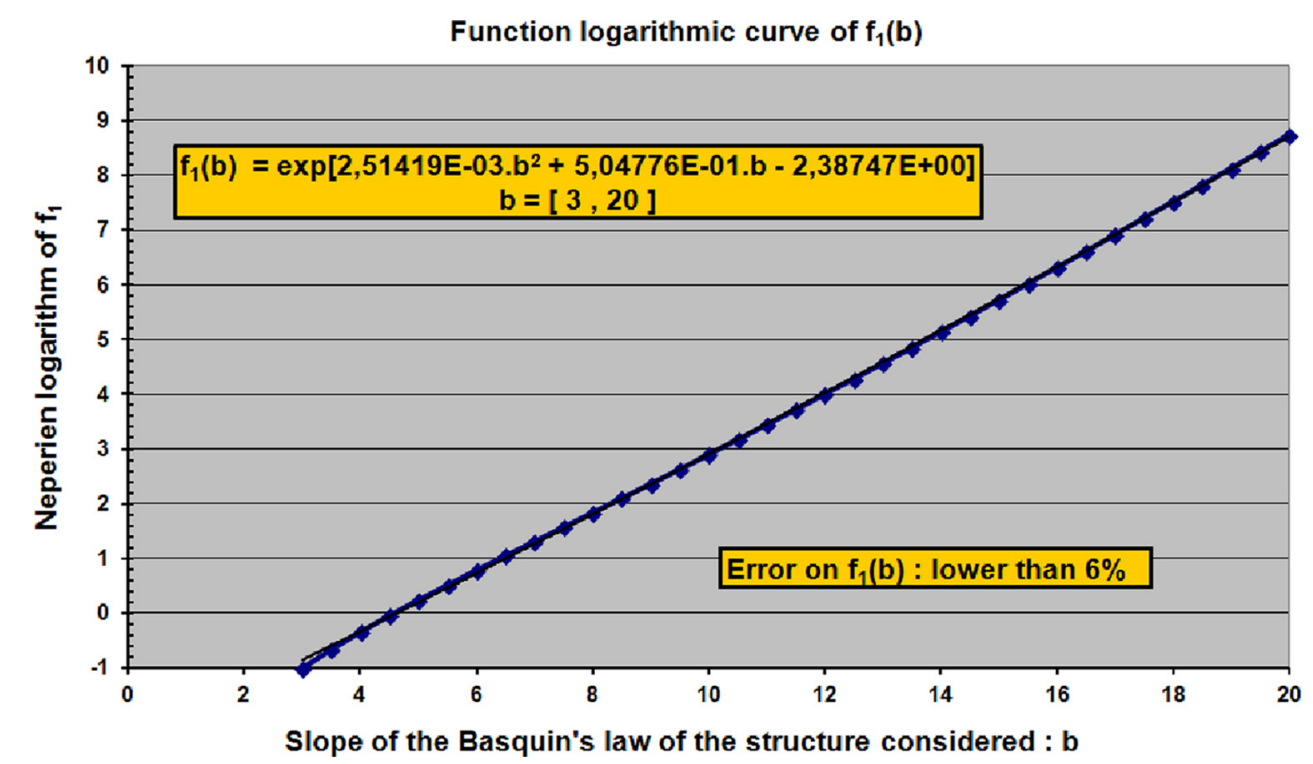

Fig. 4. Analytical expression of the $f_{1}(b)$ function, associated with calculation of spectral XFS.
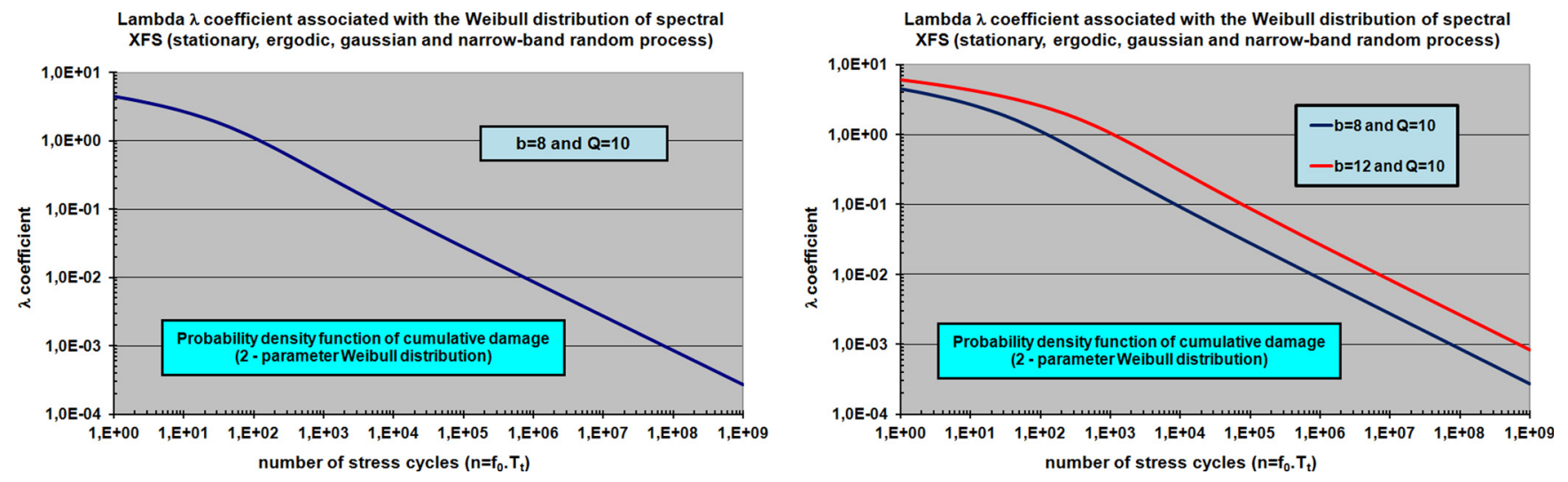

Fig. 5. Coefficient $\tilde{\lambda}$, associated with spectral XFS for $b=8$ and $b=12$.

The coefficient $\tilde{\lambda}$ obtained by dichotomy according to equation (19) is directly connected to the shape parameter $\tilde{\beta}$ of the Weibull distribution, selected to describe the statistical model of cumulative damage $D_{c}(\xi)$ over the period $T_{t}$ of stress during the random excitation process in question, since one can write:

$$
\tilde{\beta}=1 / \tilde{\lambda} \text {. }
$$

Thus in the case of electronic equipment $(b=4)$ and mechanical equipment $(b=8)$, we find that the coefficient $\tilde{\lambda}$ depends on the number $n$ of cycles associated with the stress response process, for a given value of $Q$ (usually taken to be equal to 10 in the case of customization approaches $[7,15])$. Graphically, we find that the value of this coefficient $\tilde{\lambda}$ decreases with the number of cycles $n$, for a given parameter $b$. In addition, for a given value of numbers of cycles, the greater the value of $b$ (representative of the slope of the Basquin model) increases, the higher the coefficient $\tilde{\lambda}$, as shown in the following Figure 5 .

By now injecting the value of the coefficient $\tilde{\lambda}$ into the analytic expression of the XFS provided by equation (19), it is possible to plot the relationship between the spectral XFS and the spectral FDS, conventionally used in customizing trials for a $\alpha$ given up-crossing risk. We thus obtain the following graphs.

At this stage, it should be noted that the spectral XFS is particularly relevant for taking into account the fact that the number of stress cycles $n$ in question is low, the risk $\alpha$ selected by the specifier and/or designer is low and that the Basquin slope for the material $b$ of the structure is high, as shown by the curves of Figure 6, taken from work in 2007 [6].

Based on the spectral XFS model presented above and associated with the case of narrow-band stationary Gaussian random processes, it is now possible to assess the analytical expression of the value of the risk $\alpha_{0}$ that is implicitly associated with the case of expression of the spectral FDS. Indeed, it seems important to correctly identify the up-crossing risk with which the spectral FDS concept is associated, which is very widely used in the sector for the experimental validation of products, as indeed it is today in the field of product design, which increasingly uses this technical contour to substantiate 

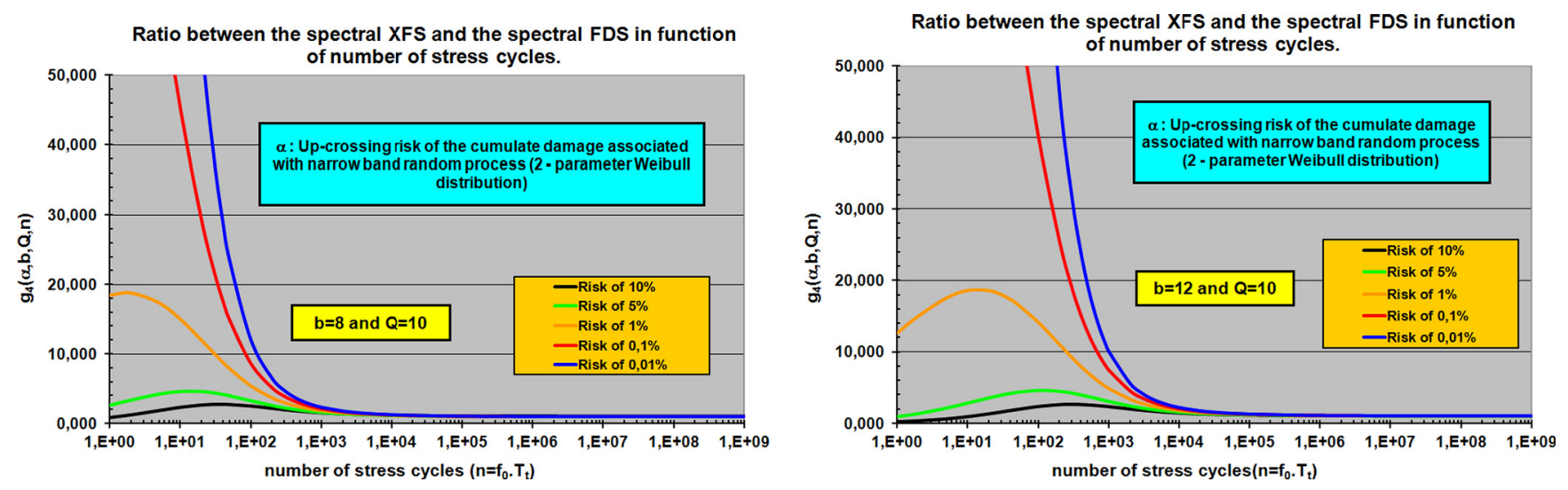

Fig. 6. Relationship between the spectral XFS and classical spectral FDS for $b=8$ and $b=12$.
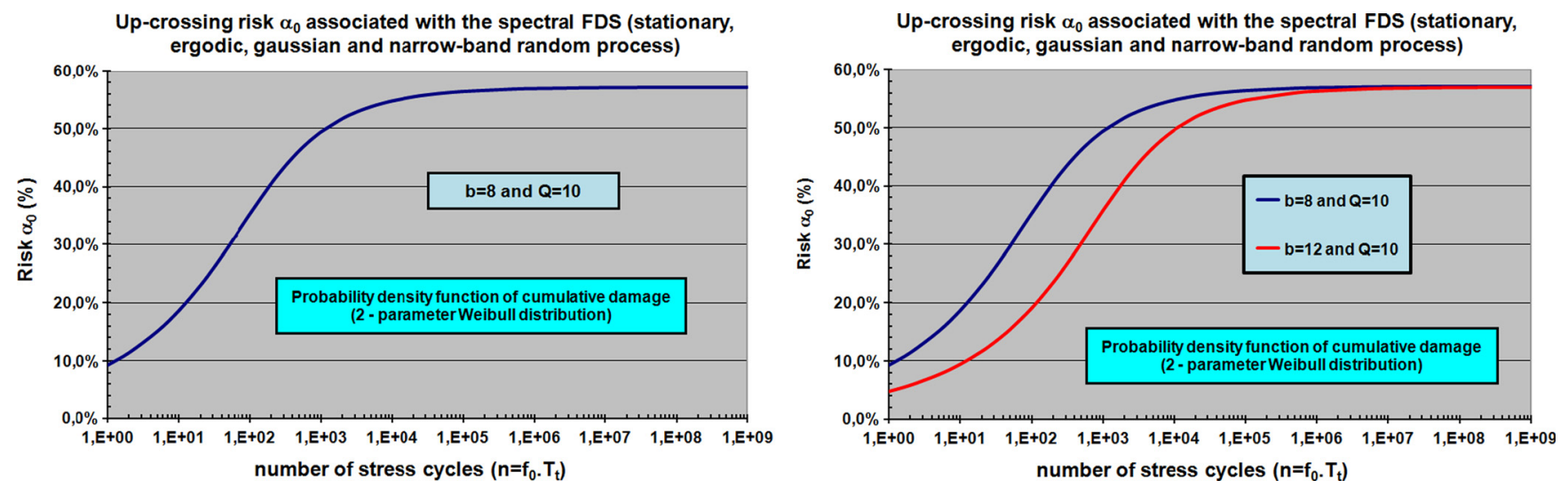

Fig. 7. Up-crossing risk, associated with the classical spectral FDS for $b=8$ and $b=12$.

the fatigue behavior of structures subjected to a vibratory environment, in terms of reliability. Due to these technical considerations, according to equations (19) and (20) we can state:

$$
\alpha_{0}=\exp \left[-\left[\Gamma\left(1+\frac{1}{\widetilde{\beta}}\right)\right]^{\widetilde{\beta}}\right] .
$$

Graphically for given values of $b$ and $Q$, the shape of the up-crossing risk $\alpha_{0}$ depending on the number $n$ of cycles shown in Figure 7 below, namely:

This therefore shows that the spectral FDS of a stationary Gaussian random excitation process is associated with an up-crossing risk $\alpha_{0}$ which is not constant and which is not equal to $50 \%$ as some authors tend to say. This risk $\alpha_{0}$ depends on the $b$ and $Q$ parameters, but especially the number of stress cycles, as seen by the structure in terms of stress response, as shown in Figure 7 above. The greater the number of cycles increases, the greater the upcrossing risk $\alpha_{0}$ increases, reaching an asymptotic value of the order of $57 \%$, in the case of structures characterized by current values of $b$ ranging from 8 to 12 , for example. We also note that for a value of given number of cycles $n$, the higher the value of $b$, the lower the risk $\alpha_{0}$, showing the need to clearly identify the nature of the critical areas of failure, the reliability of which needs to be evaluated, while controlling the associated risk.

\subsection{Calculation of the temporal XFS}

In the case of a non-stationary Gaussian process of duration $T_{t}$ characterized by a single stress time sample $z(t)$ of limited duration $T_{\text {ref }}\left(T_{\text {ref }}<T_{t}\right)$ it is no longer possible to rely on spectral approaches to assess its XFS. Although these non-Gaussian processes will always be characterized in terms of frequency by their PSD, the probabilistic structure of partial damage is only accessible by analyzing their first statistical moments, evaluated over the period of time $\left[0, T_{\text {ref }}\right]$. To do so, the temporal approach [15] (called the DBM Method, for Disjoint Block Method) implemented in the case of a stationary non-Gaussian random process consists in now analyzing the local fatigue damage of the stress process by blocks of time $T_{b}$.

To simplify, here we are placed in the case of nonGaussian random processes, which we presume to be order2 stationary (by restricting the stationarity in terms of rms values). The non-stationarity of the processes, induced for example by the vehicle speed does not constitute a difficulty per se, given that in order to overcome it, it is 
useful to classify the blocks of time data $T_{b}$, retrieved from the $\ddot{x}(t)$ non-Gaussian and non-stationary excitation process, in terms of rms values $\ddot{x}_{\text {eff }}$.

Due to these technical considerations, and after calculating using the FOH technique on each standard system the relative displacement $z(t)$ over the reference duration $T_{\text {ref }}$ of stationary non-Gaussian excitation $\ddot{x}(t)$, the responses $z(t)$ of the standard systems are as if $\ddot{x}(t)$ cut into blocks of time $T_{b}$, without any overlapping. The number of analysis blocks $N$ is then equal to the ratio $\left(T_{\text {ref }} / T_{b}\right)$ and the level of damage by partial fatigue $d$ ( $i$ ) of each block $(i=1$ to $N$ ) is estimated. The damage by partial fatigue $d(i)$ of each block of time $T_{b}$ is then evaluated in the temporal domain by equation (15) above, under Basquin's law and Miner's rule.

For each response $z(t)$ of non-Gaussian type, the sample of $N$ damage values $\{d(i), i=1$ to $N\}$ thus evaluated over time $T_{b}$ is then an $N$-random sample that can be used to characterize the first statistical moments of the damage by partial fatigue $D(\xi)$ of $z(t)$, produced over time $T_{b}$. The first two statistical moments of the random variable $D(\xi)$ are then easy to calculate and have as their expressions the following relationships in terms of average $\bar{D}$ and variance $\hat{\sigma}_{D}^{2}$, which here are two non-biased statistical estimators, namely:

$$
\begin{aligned}
& \bar{D}=\frac{1}{N} \cdot \sum_{i=1}^{N} d(i) \text { where } N=\frac{T_{\text {ref }}}{T_{b}} \text { and } \\
& \hat{\sigma}_{D}^{2}=\frac{1}{N-1} \cdot \sum_{i=1}^{N}[d(i)-\bar{D}]^{2} .
\end{aligned}
$$

The damage by accumulated fatigue $D_{c}(\xi)$ associated with the (order-2) stationary non-Gaussian and zero mean random stress process over the entire duration $T_{t}$ is then defined by equation (23) below. To do so, once again we use the Miner principle, considering an extrapolation factor over time (denoted $M$ ), which is associated with the excitation process $\ddot{x}(t)$ measured or estimated over $T_{\text {ref }}$, given that the objective is now to estimate the accumulated fatigue damage over the total period $\left(T_{t}>T_{\text {ref }}\right)$ by including its statistics.

$$
D_{c}(\xi)=\sum_{i=1}^{M} D_{i}(\xi) \quad \text { where } M=T_{t} / T_{b}
$$

$M$ : extrapolation factor over time.

The $M$ random variables representative of the damage by partial fatigue $D_{i}(\xi)$ are assumed here to be independent and identically distributed (i.i.d), which therefore allows us to apply the central limit theorem (CLT) to define the law of asymptotic probability of cumulative damage $D_{c}(\xi)$. This hypothesis, related to the use of CLT is only acceptable if the extrapolation factor over time $M$ is sufficiently large, which is a statistical validation criterion to be satisfied first of all, namely:

- if the random variables laws $D_{i}(\xi)$ are close to a normal distribution, then $(M>4)$ is sufficient;

- if the random variables laws $D_{i}(\xi)$ are moderately close to a normal distribution, then $(M>12)$ is sufficient (for symmetrical distributions around their mean);
- if the random variables laws $D_{i}(\xi)$ are not close to a normal distribution, then $(M \geq 100)$ is to be considered (case of highly asymmetric distributions, which corresponds here to our case study, since the cumulative damage by fatigue is by definition positive support.

In practice, one can be satisfied with an extrapolation factor with a minimum duration of 30 , but it would be unwise to go below, to avoid estimating irrelevant and unrepresentative fatigue damage $(1-\alpha)$ quantiles of the excitation process $\ddot{x}(t)$ in question. Therefore the asymptotic distribution of $f_{D_{c}}(y)$ accumulated fatigue damage is comparable to a normal distribution (under the assumption that the random variables $D_{i}(\xi)$ are i.i.d) centered on $\bar{D}_{c}$ and standard deviation $\hat{\sigma}_{D_{c}}$. It is thus written as follows:

$$
f_{D_{c}}(y) \underset{M \operatorname{high}}{\longrightarrow} \frac{1}{\hat{\sigma}_{D_{c}}} \cdot \varphi\left(\frac{y-\bar{D}_{c}}{\hat{\sigma}_{D_{c}}}\right)
$$

where $\varphi(y)=\frac{1}{\sqrt{2 \pi}} \exp \left(-\frac{y^{2}}{2}\right)$

where $\bar{D}_{c}=M \cdot \bar{D}$ and $\hat{\sigma}_{D_{c}}=\sqrt{M} \cdot \hat{\sigma}_{D}$ and where $M$ : extrapolation factor over time, where $\phi(y)$ represents the reduced centered normal distribution (zero mean and unit variance), often denoted $N(0,1)$ in statistics.

Due to these technical considerations, it is possible to evaluate the expression of temporal XFS, associated with a given up-crossing risk $\alpha$, based on the definition of $(1-\alpha)$ quantile of the distribution law $f_{D_{c}}(y)$ defined in equation (24) above. We then obtain the analytical expression of $(1-\alpha)$ quantile, denoted $D_{c, \alpha}$, which is homogeneous in the quantity $D_{c}$ of equation (16) of the deterministic temporal approach.

$$
D_{c, \alpha}\left(f_{0}\right)=\bar{D}_{c} \cdot\left[1+\sqrt{2} \cdot \mathrm{CV}\left[D_{c}(\xi)\right] \cdot \operatorname{erf}^{-1}(1-2 \alpha)\right]
$$

where $\mathrm{CV}\left[D_{c}(\xi)\right]=\hat{\sigma}_{D_{c}} / \bar{D}_{c}$ and $\operatorname{erf}(\mathrm{x})=\frac{2}{\sqrt{\pi}} \cdot \int_{\lambda=0}^{\mathrm{x}} \exp \left(-\lambda^{2}\right) \cdot d \lambda$

Based on the unity of an FDS which corresponds to that of a fatigue damage (dimensionless value), in relation to the standard coefficient $\left(K^{b} / C\right)$, it is then possible to write the analytical expression of the temporal XFS as follows:

$$
\begin{aligned}
& \mathrm{XFS}_{\mathrm{tp}}\left(f_{0}\right)=\frac{C}{K^{b}} \cdot D_{c, \alpha} \\
& \quad=M \cdot \bar{D} \cdot\left[1+\sqrt{\frac{2}{M}} \cdot \mathrm{CV}[D(\xi)] \cdot \operatorname{erf}^{-1}(1-2 \cdot \alpha)\right]
\end{aligned}
$$

where $\mathrm{CV}[D(\xi)]=\hat{\sigma}_{D} / \bar{D}$ and where $\left(\bar{D}, \hat{\sigma}_{D}\right)$ are defined by $(22)$, where $\mathrm{CV}[D(\xi)]$ is the experimental coefficient of variation of partial damage, associated with the random stress response process, evaluated through on standard system $\left(f_{0}, Q\right)$, for a measuring time equal to $T_{\text {ref }}$. 


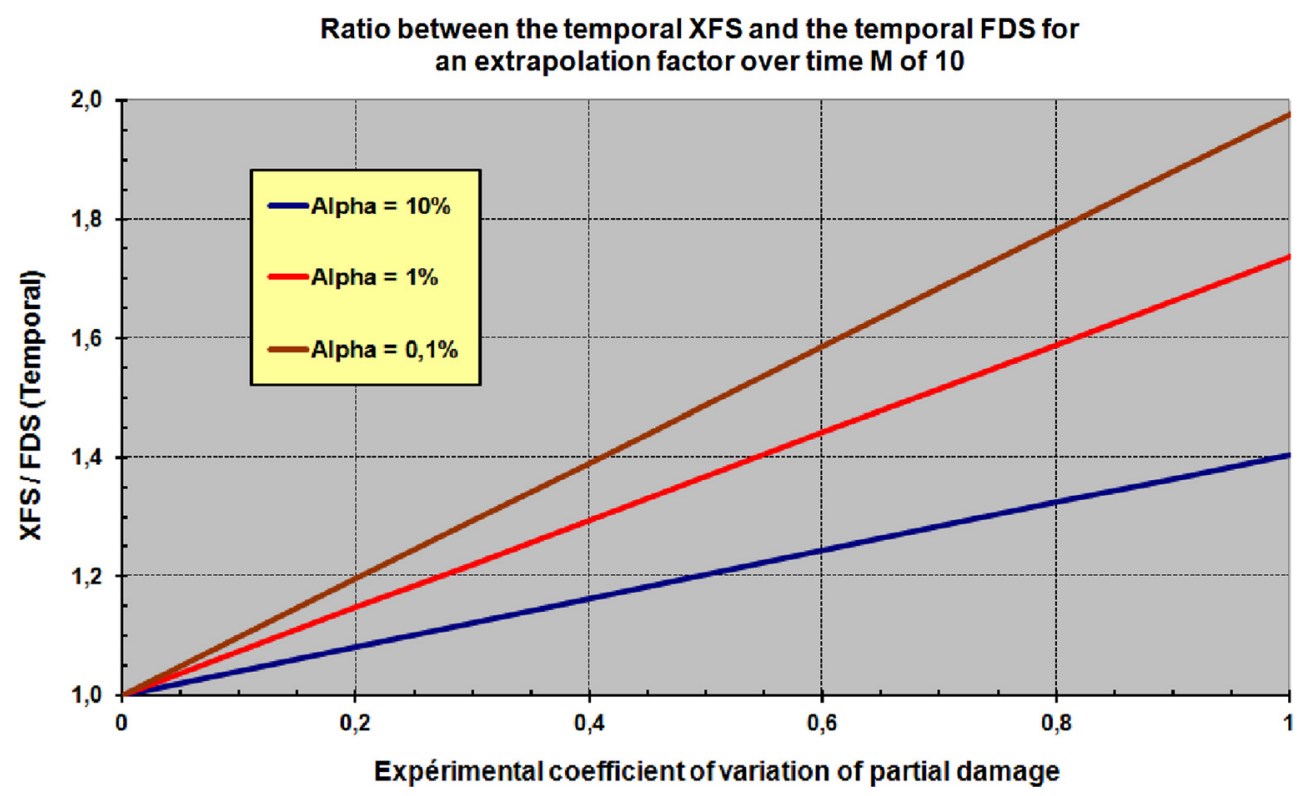

Fig. 8. $\operatorname{XFS}_{t p}\left(f_{0}\right) / \operatorname{FDS}_{t p}\left(f_{0}\right)$ pour $M=10$.

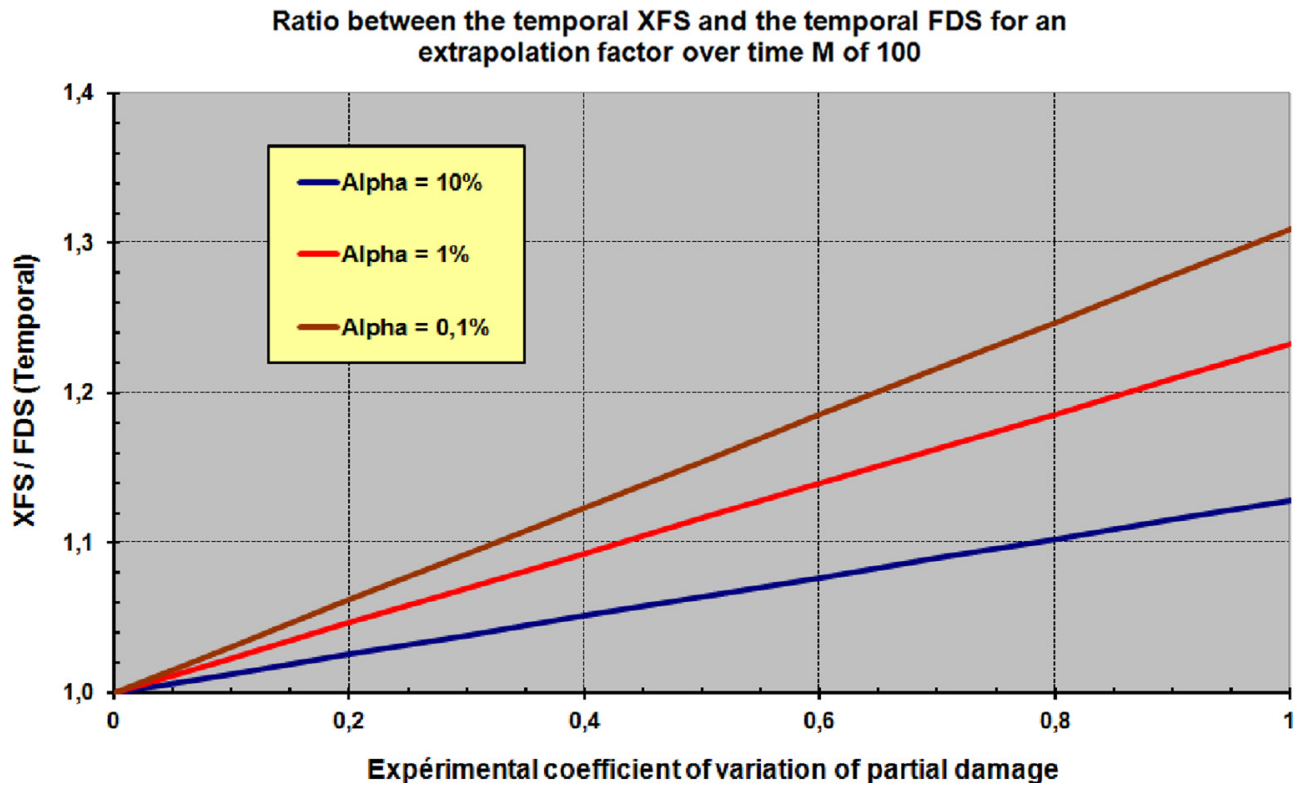

Fig. 9. $\mathrm{XFS}_{t p}\left(f_{0}\right) / \mathrm{FDS}_{t p}\left(f_{0}\right)$ pour $M=100$.

By definition, the temporal FDS noted $\mathrm{FDS}_{\mathrm{tp}}\left(f_{0}\right)$, being associated with the up-crossing risk equal to $50 \%$, we see that we can immediately write:

$$
\frac{\mathrm{XFS}_{\mathrm{tp}}\left(f_{0}\right)}{\operatorname{FDS}_{\mathrm{tp}}\left(f_{0}\right)}=1+\sqrt{\frac{2}{M}} \cdot \mathrm{CV}[D(\xi)] \cdot \operatorname{erf}^{-1}(1-2 . \alpha)
$$

where $\operatorname{CV}[D(\xi)]$ : experimental coefficient of variation of partial domage.

This shows that the ratio between the temporal XFS and the temporal FDS is a function of $\alpha$, of $M$ and of $\mathrm{CV}[D(\xi)]$ which can be represented graphically as follows for an extrapolation factor over time $M$ of 10,100 or 1000 (plotted respectively in Figs. 8-10 below).

For an extrapolation factor $M$ of given time, we see that the ratio between the temporal XFS and temporal FDS increases with the increase in the experimental coefficient of variation of partial damage CV $[D(\xi)]$ as well as with the reduction in the self-imposed up-crossing risk $\alpha$.

By drawing on the results of previous graphs, we show that for a given stationary non-Gaussian random stress process (with a given coefficient of variation of partial damage) and a given level of risk, the longer the stress 


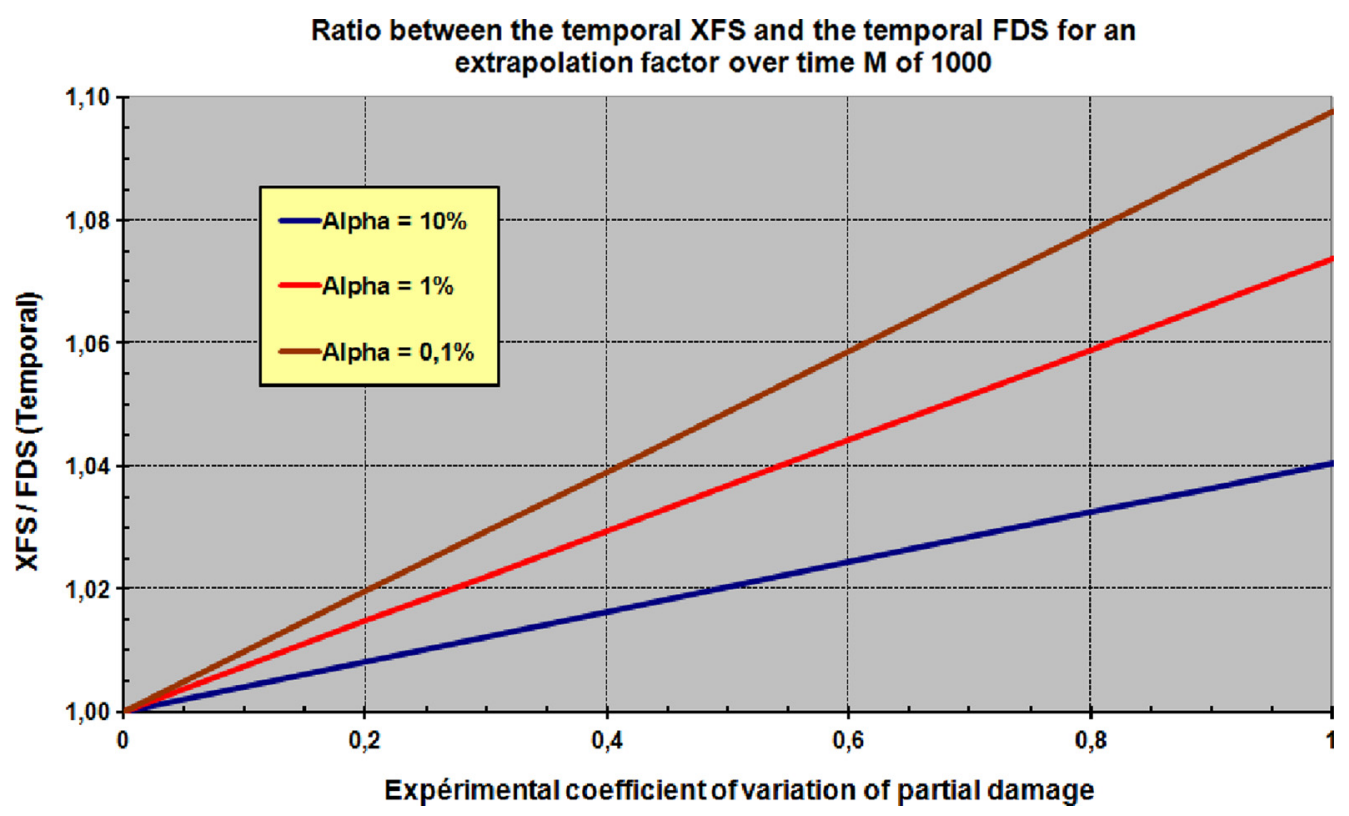

Fig. 10. $\mathrm{XFS}_{t p}\left(f_{0}\right) / \operatorname{FDS}_{t p}\left(f_{0}\right)$ pour $M=1000$.

period (the greater the increase in $M$ ), the lower the ratio between the temporal XFS and the temporal FDS.

These temporal FDS and/or temporal XFS calculation techniques are conditioned by the choice of the length $T_{b}$ of the analysis block of partial damage, associated with the non-Gaussian random process in question. The scientific bases developed previously, show that it is important to respect some basic criteria to define the optimal value of this analysis time $T_{b}$, namely:

- reduce $T_{b}$ as much as possible, in order to obtain a number $N$ (see Eq. (22)) sufficiently high to evaluate, with a good level of accuracy the first two statistical moments of partial damage $D(\xi)$. This also means increasing the extrapolation factor over time $M$ of the process (see Eqs. (23) and (24)) and to substantiate the use of the CLT to evaluate the statistical model of cumulative damage $D_{c}(\xi)$;

- increase $T_{b}$ as much as possible, to ensure the existence of a sufficient number of cycles to evaluate the partial damage that is not strongly correlated between two successive blocks, in order to satisfy the independence assumption of the previous equation (23).

These two criteria being contradictory by nature, the choice of the period $T_{b}$ of the so-called DBM Method in practice depends on the reference measurement time $T_{\text {ref }}$ and the minimum value of $f_{0}$ required.

\section{Conclusions}

The RS techniques, historically developed in terms of temporal FDS for shock phenomena, and spectral FDS for random vibration phenomena, do not make it possible to properly characterize non-Gaussian random processes in terms of fatigue damage.
To do so, temporal XFS techniques were developed and tested under the Land Weapons programs (VBCI, ARAVIS and CAESAR), highlighting their characteristics of robustness and speed of calculation.

Faced with the increased use of RS for the structural design and drafting of qualification test specifications for the equipment, these techniques of temporal XFS calculations have recently been proposed as part of AFNOR standardization work. Taking into account the lessons learned acquired by Nexter Systems on the use of these innovative approaches developed by the author in an industrial context, the members of the AFNOR Group have adopted them as part of NF X50-144-3 [16], for their ability to positively expand RS techniques to very widespread processes in industry, and so far poorly characterizable by conventional temporal FDS and/or spectral FDS approaches.

As illustrated by this standard, these tools (temporal XFS) for characterizing fatigue damage, with an imposed up-crossing risk, thus make it possible to greatly expand the scope of the calculation methods of RS, by now covering the case of non-Gaussian processes (whether narrow-band or broadband).

The non-Gaussian nature of the processes now being taken into by these temporal approaches of a stochastic nature, it is no longer necessary to use the deterministic temporal approaches, described in Section 3.2, in particular since they are non-conservative.

\section{References}

[1] J.F. Imbert, Analyse des Structures par Eléments FinisCépadues Editions

[2] C. Lalanne, Vibrations et chocs mécaniques- Hermes Science publications, copyright Lavoisier, 1999: Tome 5-Élaboration des spécifications 
[3] J.S. Bendat, Principles and applications of random noise theory, John Wiley \& Sons, New York, 1958

[4] R.W. Hamming, Numerical methods for scientists and engineers, 2nd edn, ISBN 0486652416

[5] D.O. Smallwood, An improved recursive formula for calculating shock response spectra, shock and vibration Bulletin: Proceedings of the 51st Symposium on Shock and Vibration, San Diego, California, 1980

[6] B. Colin, La probabilisation des SDF: une réponse à la comparaison des sévérités d'environnement déterministe et stochastique, en termes d'endommagement par fatigue, 2527 septembre 2007, ASTELAB 2007

[7] NORMDEF 0101, Guide d'application de la démarche de personnalisation en environnement mécanique, DGA 2009, Edition 01, Julliet 2009.

[8] B. Colin, Approche stochastique de la Fatigue vibratoire: comparaison entre les méthodes Peak-Valley et Rainflow Counting, Rev. Métall., 2012, DOI:10.1051/metal/2012024

[9] M.A. Miner, Cumulative damage in Fatigue, J. Appl. Mech. 67 (1945) A159-A164

[10] S.O. Rice, Mathematical analysis of random noise, Bell Labs Tech. J. 23 (1944) 282-332
[11] A. Preumont, Random vibration and spectral analysis, Kluwer Academics Publishers, Dordrecht, The Netherlands, 1994

[12] C. Lalanne, Vibrations et chocs mécaniques- Hermes Science publications, copyright Lavoisier, 1999: Tome 2-Chocs mécaniques

[13] B. Colin, La probabilisation des SRE: une réponse à la comparaison des sévérités d'environnement déterministe et stochastique, en termes de contraintes extrêmes, 25-27 Septembre 2007, ASTELAB 2007

[14] Stephen H. Crandall, W.D. Mark, Random vibration in mechanical systems, Hardcover, Academic Press Inc., New York, 1963

[15] B. Colin, Characterization of random Gaussian and nonGaussian stress processes in terms of extreme responses, in: Matec Web of Conferences, EDP Sciences, DOI:10.1051/ matecconf $/ 20152006001$.

[16] AFNOR, Norme NF X50-144-3, Démonstration de la tenue aux environnements-Conception et réalisation des essais en environnement-Partie 3: Application de la démarche de personnalisation en environnement mécanique, 2014

Cite this article as: B. Colin, Characterization of random Gaussian and non-Gaussian stress processes in terms of vibration fatigue, Mechanics \& Industry 18, 809 (2017) 